\title{
Idéologies et pratiques managériales: du taylorisme à la précarisation subjective des salariés
}

Danièle Linhart*

Résumé: Cet article développe l'idée que tout nouveau modèle capitaliste d'organisation du travail produit une rhétorique idéologique et morale destinée à légitimer une forme de mise au travail qui s'avère contradictoire à l'essence des démocraties politiques, à savoir que chaque individu s'appartient à lui-même. Taylor et Ford ont fait de grands efforts pour convaincre l'opinion publique qu'il n'y avait pas de conflits d'intérêts entre les ouvriers et leurs patrons, tout en attaquant systématiquement leurs ressources dans le cadre d'un rapport de forces bien réel. Le management moderne français suit la même voie et cherche à séduire et convaincre les salariés tout en développant une stratégie de précarisation subjective fondée sur une politique de changement perpétuel pour les déstabiliser.

Mots-clés: Taylor, Ford, idéologie, management moderne, précarisation subjective.

\section{Présentation}

e management français s'est fixé pour mission de métamorphoser ses salariés de manière à les rendre plus adaptés aux nouvelles donnes de la concurrence économique et plus récemment aux contraintes du capitalisme financier. Car il est dans son ensemble convaincu qu'il a en héritage de la période antérieure, notamment celle des Trente Glorieuses, une population de salariés plus rétive, plus rebelle, plus difficile à manager que celle des autres pays occidentaux concurrents. II a cette conviction, partagée par une bonne partie de l'opinion publique, que les salariés français sont enclins à se mettre dans les rails de la contestation, de la confrontation, voire de la lutte des classes, qu'ils sont farouchement arc boutés autour de leurs acquis, avec une solide propension à faire grève ou encore qu'ils n'en font qu'à leur tête, persuadés d'avoir toujours raison (selon la logique de l'honneur professionnel, dont parle Philippe d'Iribarne, 1989). II faut dire que les salariés français cumulent historiquement bien des travers du point de vue de leurs employeurs; la France est un pays où le parti communiste a représenté une réelle force politique dans l'après guerre, où le syndicalisme (particulièrement la CGT) était fortement conflictuel, où le travail cristallise plus vivement qu'ailleurs les passions ( Davoine \& Méda, 2008), représente un enjeu politique, symbolique, plus prégnant, et où la réduction de la durée du travail est parmi les plus fortes. Il y a parmi les décideurs politiques depuis les années 80 une réelle tendance à considérer que les Français sont enclins à en faire le moins possible

\author{
* Directrice de \\ recherches émérite \\ au Centre national \\ de la recherche \\ scientifique \\ (CNRS). Laboratoire \\ Genre, Travail, \\ Mobilités, Centre \\ de Recherches \\ Sociologiques et \\ Politiques de Paris. \\ Université de Paris \\ 10 - Nanterre. \\ <Daniele.linhart@ \\ gtm.cnrs.fr>.
}


1. Yves Montand dénonçait l'égoïsme des Français qui refusent de consentir les efforts pour préserver le pays de d'un déclin économique. et qu'ils doivent réviser sérieusement leur rapport au travail. Cela a commencé avec l'émission Vive la crise animée par Yves Montand ${ }^{1}$ et relayée par Libération (le 22 février 1984 ), s'est poursuivi avec l'allocution du premier ministre Raffarin au Québec incitant les Français à se remettre au travail (La France ne saurait être un pays de parcs d'attraction), plus tard Nicolas Sarkozy reprendra cette thématique de la nécessité de réhabiliter la valeur travail. Le phénomène des 35 heures a largement amplifié cette perception d'un pays où on se laissait aller à des attitudes frisant la paresse. L'importance du secteur public et du nombre des fonctionnaires, de même qu'un code du travail et une prise en charge sociale encore protecteurs renforce cette perception d'une population de salariés privilégiés et même souvent trop assistés.

Pour que la France garde sa place au rang des nations puissantes, qu'elle conserve ses entreprises, et ses emplois, il faut donc inaugurer une autre ère. Et produire d'autres salariés, dotés d'un autre rapport au travail, d'une autre vision du monde. Car les transformations économiques mondiales impliquent impérativement des manières de produire biens et services qui intègrent des exigences de flexibilité, mobilité, réactivité, loyauté, réceptivité aux consignes, acceptation de prise de risques, c'est-à-dire autant de remise en cause des formes antérieures de mobilisation au travail. Pour asseoir un nouveau mode de mise au travail, il convient alors de formater d'une manière bien spécifique les salariés français, et ce d'autant plus qu'ils sont a priori plus éloignés que bien d'autres populations de salariés, de ces qualités nécessaires, de cette forme d’employabilité recherchée.

L'analyse des différents modèles de mise au travail au cours de l'histoire nous enseigne que toute nouvelle forme d'organisation du travail nécessite un type particulier de salariés, de mode de vie et même de société et qu'elle s'accompagne d'une rhétorique idéologique et morale spécifiques légitimant le modèle.

\section{Le salariat: une enclave dans toute société démocratique. Le discours de légitimation du taylorisme et du fordisme}

Pierre Rosanvallon dans son cours Le désenchantement de la démocratie: histoires et formes d'un sentiment au Collège de France (2004), cite Stendhal. Celui-ci écrivait en effet dans son pamphlet «Un nouveau complot contre les industriels», publié en 1825:

Pendant que Bolivar affranchissait l'Amérique, pendant que le capitaine Parry s'approchait du pôle, mon voisin a gagné dix millions à fabriquer du calicot; tant mieux pour lui et pour ses enfants. Mais depuis peu il fait faire un journal qui me dit tous les samedis qu'il faut que je l'admire comme un bienfaiteur de l'humanité. Je hausse les épaules. Les industriels sont peut-être des gens honorables mais ils ne sont pas des gens héroïques. 
Stendhal identifie de façon magistrale cette propension des industriels, des employeurs à se faire reconnaître comme des bienfaiteurs de l'humanité, comme œuvrant pour le bien général, pour l'intérêt commun.

Cette orientation a accompagné toute la mise au travail capitaliste. Car il faut justifier ce fait inconvenant dans une démocratie politique que des individus soient contraints de renoncer au libre usage d'eux-mêmes, à leur libre arbitre, pour se soumettre à une logique, une volonté qui leur est extérieure. Le temps (de travail) du salarié appartient en effet à son employeur qui l'a acheté dans le cadre du salariat. Le salarié doit accepter de se déposséder du libre usage de son propre temps, il doit accepter de travailler en fonction des consignes et objectifs fixés par son employeur. Comment refuser de voir qu'il y a là un contentieux inépuisable et que l'on se trouve, avec ce contrat salarial de dépendance, dans une véritable enclave inappropriée à la logique démocratique qui veut que tout individu s'appartient ? Chaque personne au travail a ses propres intérêts sur lesquels elle doit veiller. Chaque personne a également un point de vue sur la manière dont elle souhaiterait s'y prendre pour faire son travail, en fonction de son métier, de sa professionnalité, de son expérience, de sa sensibilité, de sa personnalité, de son rapport au monde, de son éthique personnelle. Mais, l'employeur veut que les personnes qu'il paye, dont il a acheté le temps de travail et les savoirs, travaillent de façon à atteindre la productivité, la qualité et donc la rentabilité les plus fortes possibles.

\subsection{Taylor et le sens de l'intérêt général}

Cela choque, sans doute maintenant, que l'on puisse penser à Taylor (dont le nom est associé à l'éclatement des métiers, à la déqualification, l'hyperspécialisation, au travail en miettes et à l'autoritarisme ) comme à un bienfaiteur de l'humanité.

Mais Taylor s'est donné beaucoup de mal pour justifier sa démarche et lui donner les allures d'une réforme majeure qui associe progrès économique et progrès social. ॥ est vrai que l'organisation scientifique du travail a permis des augmentations substantielles de productivité, ouvrant une consommation plus abondante à moindre prix pour le marché américain auquel les ouvriers avaient accès grâce à des rémunérations plus élevées. Mais avec une terrible contrepartie qui était une dépossession de la maîtrise du travail, car comme l'écrit avec enthousiasme le deuxième préfacier du livre écrit par Taylor (1956) lui-même et intitulé, La direction scientifiques des entreprises:

[...] ce que Taylor nous demande, c'est d'abandonner temporairement chaque jour, cette indépendance d'esprit, d'accepter de faire partie d'un groupe dans lequel notre travail est forcément limité 
et spécialisé, pour produire mieux et pouvoir profiter, le reste du temps, à notre guise, de cette production supplémentaire, qui est le résultat de notre effort (Taylor, 1956: 12).

L'invention de Taylor consiste à transformer des ouvriers de métier en exécutants et en consommateurs. Il est intéressant d'analyser comment il a fait la promotion de sa méthode en développant des points forts et bien réels, lui permettant de présenter comme un pas en avant, un progrès décisif ce qui correspondait de fait à une attaque en règle du registre professionnel des ouvriers, les plongeant dans un état de soumission et de dépendance hiérarchique inouï pour l'époque. On tentera de faire de même pour Ford avant de soumettre le modèle managérial moderne au même exercice.

Dans l'argumentation de Taylor, on trouve plusieurs registres. D’abord celui de la paix sociale, de la réconciliation entre ouvriers et patrons. II part d'un constat, celui de l'existence d'une lutte stérile entre patrons et ouvriers. Il épingle en effet la flânerie systématique des ouvriers, qui n'est que la conséquence d'une mésentente et qui suscite une répression patronale; il se veut juste et mesuré dans l'analyse qu'il en fait:

La plus grande part de flânerie systématique est accomplie par des ouvriers qui ont pour objectif raisonné de maintenir leurs employeurs dans l'ignorance de la qualité de travail qu'ils peuvent normalement effectuer (Taylor, 1956: 29).

En effet, si le patron découvre que l'ouvrier est «capable de faire plus de travail qu'actuellement, il trouvera tôt ou tard un moyen de l'obliger à le faire en n'augmentant pas son gain ou en l'augmentant très peu». Taylor comprend que les ouvriers se situent dans un rapport de force, un conflit d'intérêts bien réel, bien concret avec leur patron. Et c'est cela qu'il veut éradiquer. On voit très précisément qu'il cherche à se substituer aux ouvriers pour définir à leur place quels sont leurs intérêts. II ne met pas en cause leur honnêteté («ceci est le point de vue honnête de l'ouvrier moyen dans pratiquement toutes les professions») mais il veut montrer qu'ils sont dans l'erreur, qu'ils n'ont pas compris, qu'ils se trompent: ce préjugé adopté par la classe ouvrière est «fallacieux» écrit-il (idem: 35). II ne prend évidemment en considération qu'une dimension de la réalité, le fait que plus de productivité peut entrainer une croissance et plus d'emploi«chaque fois qu'il y a eu augmentation de la production... il y a eu plus de travail pour un plus grand nombre d'hommes» (ibidem: 37). Mais il fait l'impasse sur le fait que la flânerie est une arme ouvrière dans le cadre d'un rapport de force. Car ce que veut précisément éradiquer Taylor c'est la possibilité pour les ouvriers de s'opposer, d'imposer quoi que ce soit au patron. II veut y arriver au nom ce qu'il met en avant comme l'intérêt commun, c'est à dire la progression des emplois et de la consommation pour tous. II veut montrer que les ouvriers méconnaissent leurs véritables intérêts, et font obstacle au développement de la consommation pour tous. II y a va de la morale: 
Toute association d'hommes qu'il s'agisse d'un groupe d'ouvriers ou d'un groupe de capitalistes et d'industriels, ou tout autre groupe quelconque, tout groupe, dis-je, qui délibérément, restreint la production d'une industrie vole, par ce fait même; la peuple - écrit-il (Taylor, 1956: 41).

Et il enfonce le clou:

Je veux faire comprendre que je crois que c'est un crime pour un industriel de limiter la production pour maintenir les prix et c'est également un crime pour l'ouvrier d'agir de même pour toute autre raison (Taylor, 1956: 41).

Sa grande idée, sa «trouvaille» pourrait-on dire, c'est d'interposer la science entre des ouvriers et les patrons qui n'adoptent pas une attitude, une stratégie raisonnables même de leurs points de vue. La science permettra de résoudre le contentieux au profit de tous. "C'est un moyen très efficace et très sûr de rendre les hommes plus efficients qu'ils ne le sont actuellement et ceci sans leur donner une plus grande charge de travail» (ibidem: 39).

On voit à l'œuvre nombre de registres qu'on retrouvera chez Ford et les adeptes du management moderne: la morale, l'objectivité, la scientificité, la neutralité, le bien commun, l'humanité dans une même unilatéralité, c'est-à-dire en niant toute une partie de la réalité, le savoir, les intérêts divergents, le rapport de force, la nécessité pour les ouvriers de disposer de contrepouvoirs pour ne pas subir, dans un état de dépendance totale, le diktat patronal. Taylor, s'évertue ainsi à nier la dimension politique du travail pour n'en retenir que la dimension technique, ou morale, afin de créer les conditions unilatérale d'un consensus. Car il passe sous silence ce fait que la science sera mise en œuvre par le patron. Il a décidé, en effet, que ce serait au patron de mobiliser cette science, ce qui suppose de déposséder les ouvriers de leur métier. Or, détenir un métier permet d'imposer des tarifs et de faire obstacle à la volonté du patron. La question est d'importance et reste d'actualité: celui qui connaît le travail est en position de s'imposer; il dispose d'un atout de taille que précisément l'organisation scientifique du travail veut éradiquer, soi-disant pour le plus grand bien de tous, au nom de la morale et grâce à la science qui est pure objectivité et neutralité.

La «science» implique de fait une nouvelle «répartition de la responsabilité du travail entre la direction et les ouvriers plus équitable que celle que nous constatons dans les modes courants de direction» (ibidem: 48). II s'agit en fait d'un pur transfert de la responsabilité des ouvriers vers celle de la direction. Ce qui n'était pas équitable auparavant car présenté comme unilatéral, le devient dès lors que cette fois ce sont les patrons qui détiennent à eux seuls cette responsabilité que leur confère l'application 
de la science. La direction détenant le monopole du savoir va instruire les ouvriers pour les guider dans leurs tâches, et cela s'appellera la coopération.

Et, preuve, selon de Taylor, de l'honnêteté et l'impartialité du système, la suppression de la flânerie permet une augmentation de salaire de 30 à 100\%. En permanence, dans son livre, Taylor revient sur la nécessité de concilier les intérêts de toutes les parties prenantes,

\footnotetext{
il est en effet sage de dire que l'on ne doit pas prendre en considération les systèmes de direction qui ne donnent pas satisfaction d'une façon permanente à la fois aux patrons et aux salariés, qui ne mettent pas en évidence le fait que les intérêts des deux parties sont identiques et qui n’amènent (Taylor, 1956: 54).
}

Il ne s'agit pas de critiquer en soi l'idée d'une tentative de rapprochement, d'une recherche d'équilibre entre les intérêts et les valeurs des uns et des autres. Mais pour concilier, réconcilier, trouver des équilibres et donc passer des compromis, il faut accepter l'idée bien ancrée dans le réel, d'une divergence, d'un antagonisme. Or tout l'art et l'argumentation de Taylor vise à rendre cette idée amorale, et de la présenter comme scientifiquement fausse. Taylor se place au-dessus de la mêlée, et veut désamorcer toute critique, toute contestation possible.

Pourtant, ce qu'il met en place est une machine de guerre redoutable contre les ouvriers. Ils les dépossèdent sciemment et systématiquement de ce qui constitue leur force, leur identité, et leur pouvoir, à savoir leur métier, et leurs connaissances. II a, à l'évidence, choisi son camp. Son approche fonde les bases d'une domination patronale sans appel.

Devant les membres de la commission d'enquêtes de la Chambre des représentants américaine (il a été accusé par les syndicats de casser les métiers), il consacre beaucoup de temps à expliquer que la réforme organisationnelle qu'il préconise a des effets équivalents sur les ouvriers et la direction, qu'elle leur impose des contraintes d'une nature comparable. Ces passages sont particulièrement importants et éclairants, car ils montrent bien à quel point il est décisif pour Taylor de parvenir à faire accepter comme fair, c'est-à-dire juste, honnête, cette dépossession des ouvriers qui correspond à une nouvelle répartition des missions, des responsabilités et du travail entre les ouvriers et leur direction, extrêmement exigeante pour cette dernière.

Le quatrième principe de direction scientifique est peut-être le plus difficile à comprendre pour la moyenne des gens. II consiste en une division presque égale du travail dans l'entreprise entre l'ouvrier d'un côté, et la direction de l'autre. C'est-à-dire que le travail qui, dans I'ancien système, est accompli pratiquement en totalité par 
l'ouvrier est, dans le nouveau, divisé en deux parties: I'une de ces parties est délibérément prise en charge par ceux qui font partie de la direction... Dans un atelier, quand on dirige l'entreprise suivant le nouveau système, il n'y a pratiquement pas un seul acte accompli par l'ouvrier qui ne soit pas précédé et suivi par quelque acte accompli par quelqu'un se trouvant du côté de la direction (Taylor, 1956: 89).

Il allègue à plusieurs reprises que ces tâches imposées aux directions représentent une contrainte lourde qu'ils n'acceptent pas si facilement:

Invariablement, nous constatons une très grande opposition de la part de ceux qui font partie de la direction à accomplir leurs nouveaux devoirs et comparativement une très faible opposition de la part des ouvriers à coopérer en accomplissant leurs nouvelles tâches (Taylor, 1956: 82).

On trouve un hymne à l'abstraction:

L'homme qui se sert de règles à calcul, qui connaît la science de la coupe de métaux, bien qu'il n'ait jamais vu un travail donné, peut faire exécuter le travail mieux que ne le fait un mécanicien expérimenté qui a été spécialisé pendant de nombreuses années dans I'usinage des pièces de cette machine particulière (Taylor, 1956: 184).

Sur la base d'arguments psychologiques, il met même en garde contre le travail en équipe:

Quand des ouvriers travaillent en équipe et ne sont pas considérés comme des travailleurs indépendants les uns des autres, ils perdent ambition et initiative. Une analyse poussée a montré que quand des ouvriers sont réunis en équipe, chaque membre du groupe devient moins efficient que quand son ambition personnelle est stimulée (Taylor, 1956: 142).

Se préoccuper du bonheur des ouvriers, prétendre à un système équitable, juste et orienté vers le bien commun, invoquer la science, l'abstraction, l'objectivité, la neutralité, dévaloriser les connaissances acquises grâce à l'expérience, miser sur l'ambition personnelle, ces attitudes ne sont pas sans rappeler bien des allégations, argumentations accompagnant le management moderne. Loin de se présenter comme le consultant qu'il était alors au service des directions d'entreprise, Taylor se positionne devant les membres de la commission d'enquête et devant l'opinion publique qui lira son livre, comme un homme de science, bienfaiteur et préoccupé de la paix sociale et du bien être commun, un homme qui a mis le travail à la portée de tous. Faisant l'impasse 
sur le fait qu'il a inversé le rapport de force entre les ouvriers et leur patron, en transférant vers ce dernier le savoir ouvrier, permettant ainsi une domination qu'il voulait absolue puisqu'il a inscrit la contrainte et le contrôle dans la définition même des tâches. L'ouvrier est désormais pris en main, il est agi par l'organisation du travail (agi et contrôlé par elle). Le travail pourra se dérouler ainsi uniformément indépendamment des états d'âme, des états d'esprits des savoirs, de l'expérience des ouvriers. II ne dépendra que de la conception abstraite réalisée par des ingénieurs. La dimension cognitive du travail a changé de camp, le travail appartient désormais à la direction et ceux qui travaillent pour elle.

\title{
1.2 Ford: un pas de plus dans la mauvaise foi. L'enchaînement des contradictions
}

Henry Ford introduira les premières chaines de montage dans l'industrie pour la production des automobiles, lui aussi au nom de la prospérité américaine. Lui aussi écrira pour propager son modèle et le légitimer. Dans «Propos d'hier et d'aujourd'hui» (1926) notamment, il affirme

\begin{abstract}
Chaque fois que vous tolérez qu'un ouvrier produise moins que ce que son salaire représente, vous encouragez la baisse de son salaire réel et vous faites en sorte qu'il lui sera encore plus difficile de gagner sa vie. Vous ne sauriez causer plus grand tort à un ouvrier qu'en lui permettant de tirer au flanc. La raison en est évidente, moins un homme produit et moins nombreux seront ceux qui auront besoin du fruit de son travail (Ford, 1926: 134).
\end{abstract}

Comme son prédécesseur, il se pose en démocrate respectueux des droits individuels, soucieux du bien-être de ses ouvriers, de la prospérité économique tout en se présentant comme un homme d'industrie, rationnel, qui veut «en avoir pour son argent». II lui faut être exigeant vis à vis de ses ouvriers, veiller à ce qu'ils soient efficaces et rentables en permanence, tout en prétendant trouver des règles du jeu qui satisfassent toutes les parties. II se veut «fair» (honnête, juste) comme prétendait l'être Taylor dans les pas duquel il s'inscrit. Mais il fera encore progresser la domination que l'employeur exerce sur ses ouvriers. Dans deux sens, en empiétant sur leur vie privée par un contrôle qu'il prétend paternel mais qui n'en est pas moins impérieux; et en accentuant le contrôle de leur rythme de travail par l'instauration des chaines de montage. C'est surtout ce dernier aspect que l'on a retenu et il l'a lui-même bien mis en évidence dans ses écrits.

Notre premier progrès dans le montage consista à apporter le travail à l'ouvrier, au lieu d'amener l'ouvrier au travail. Aujourd'hui, toutes nos opérations s'inspirent de ces deux principes. Nul homme ne doit 
avoir plus d'un pas à faire; autant que possible, nul homme ne doit avoir à se baisser (Ford, 1929: 90).

Avec la chaîne, instaurée en 1913, les ouvriers sont fixés sur place et ce sont les pièces qui défilent selon un ordre séquentiel, dans un flux ininterrompu, pour être travaillées. Cette innovation majeure implique une réorganisation de l'entreprise car il faut assurer une standardisation et homogénéisation de toutes les pièces qui entrent dans l'activité de montage. Le rythme devenait une obsession pour le personnel et la maladie nerveuse qui en découle était appelée «fordite» par les ouvriers. (Ce que nous appelons le stress de nos jours).

On observe alors une véritable répulsion pour ce nouveau mode de travail qui se concrétise dans un turn-over phénoménal de 380\%. En effet, les journalistes de l'époque rapportent que les ouvriers qui attendaient dans de longues files d'attente d'être embauchés dans l'usine, quittaient les chaines au bout d'un laps de temps très court, en hurlant que c'était des conditions de travail intenables et qu'il fallait être fou pour accepter de travailler dans ces conditions. Ce turn-over pose des problèmes de régularité de la production et coûte cher à l'entreprise et il faut trouver absolument une parade pour stopper l'hémorragie.

La véritable parade sera trouvée dans le niveau de salaire qui correspond à une compensation évaluée comme suffisante. Le 5 janvier 1914, Ford décide, en effet, de doubler la paie, de passer d'une rémunération d'environ 2,5 dollars pour une journée de neuf heures à 5 dollars pour huit heures.

Cette stratégie, destinée à fidéliser une main-d'œuvre malgré des conditions de travail jugées à l'époque très pénibles voire insupportables, sera niée en tant que telle. Henry Ford écrit en effet:

Après bien des années d'expérience nous n'avons pas constaté que le travail répétitif fut préjudiciable à l'ouvrier. Le fait est qu'il semble même entraîner une meilleure santé, physique et mentale que le travail non répétitif. Si les hommes n'aimaient pas leur travail, ils partiraient (Ford, 1929: 139).

Car ce que cherche Ford c'est à mettre en avant une philosophie, une théorie économique à visée universelle. Dans Propos d'hier et d'aujourd'hui, il écrit en effet:

L'industriel qui rémunère le travail de ses ouvriers au plus bas et qui en échange de l'argent de ses clients ne verse que le salaire minimum, a un comportement identique à celui du maçon qui ne pose que la moitié des briques qu'il pourrait poser. Mais plus d'un industriel croient sincèrement qu'ils paient, en salaire, le maximum 
de ce que leurs entreprises peuvent supporter. C'est peut-être vrai mais personne ne sait ce qu'il peut payer tant qu'il n'a pas essayé. En 1915, nous avons fait passé chez Ford le salaire horaire de deux dollars et 45 cents à 5 dollars minimum, par jour. C'est à ce moment que nos affaires ont pris de l'expansion car ce jour là nous nous sommes créés un grand nombre de clients pour nos automobiles et en second lieu nous avons à découvrir tellement de manière d'économiser sur les coûts que nous avons été très vite en mesure de baisser les prix. Ce qui était une pratique visant à faire accepter des conditions drastiques aux ouvriers devient un principe économique de prospérité et moralité (Ford, 1992).

John Bellamy Foster relate à ce propos une anecdote publiée à l'époque dans The Nation, et rapportée par Keith Sward (1968):

En 1923, deux grands opérateurs de Wall Street en vinrent à évoquer ce Barnum industriel. "Ford parle comme un socialiste", dit l'un d'eux. "Certes, mais il agit comme l'un des nôtres", lui réplique calmement l'autre, "et il s'en tire très bien comme cela".

On peut en effet considérer que les nombreux écrits et discours de Henry Ford mettent en scène d'une façon très particulière une réalité qui a sa propre logique. On est dans une logique de story telling, comme celles qu'analyse Christian Salmon (2007).

Henry Ford a considérablement augmenté les salaires pour fidéliser sa main-d’œuvre alors que l'organisation du travail qui lui est imposée est répulsive, mais, à ce tarif là, il veut être certain d'en avoir pour son argent, il veut s'assurer que les ouvriers méritent leurs salaires, non seulement en se soumettant aux contraintes de la chaine, mais en adoptant un mode de vie, qui correspond bien à ces contraintes et qui assurera un bon état de leur force de travail. Ford veut procéder ainsi non seulement à une standardisation des pièces qui entrent dans la production mais à une standardisation également de la vie de ses ouvriers et initie un contrôle étroit de leur vie. II dit dans My philosophy of industry, an authorized interview by Fay Leone Faurote (1929), que pour qu'une production tourne bien il faut que les outils et l'usine soient propres, les indicateurs précis, les méthodes elles aussi précises et il faut aussi des gens, avec une vie domestique qui tourne bien, des gens qui pensent correctement, qui vivent correctement, normalement.

Et pour cela, il met sur pied un Département de sociologie où des inspecteurs sont chargés d'aller vérifier au domicile privé des ouvriers qu'ils respectent bien les conditions d'hygiène et de moralité, qui sont nécessaires pour mériter le five dollars. Sinon, le travailleur reçoit uniquement un salaire de 2,5 dollars. Ainsi, durant les deux premières années d'application, $28 \%$ des travailleurs se sont vu refuser l'accès au pro- 
gramme. En 1917, l'équipe des inspecteurs est composée de 52 inspecteurs réguliers et 14 membres spéciaux. L'idée qu'un mode d'organisation industriel implique mode de vie spécifique de la part des employés est particulièrement bien concrétisée par Ford. II avait pour ambition de faire émerger un ouvrier nouveau, adapté physiquement à ses chaines de montage, grâce à une épouse qui doit bien le nourrir, le tenir et savoir faire des économies pour acheter la voiture produite dans ses usines. Les inspecteurs prodiguaient des conseils en matière diététiques, ils proposaient des menus types concoctés par Henry Ford lui-même qui était végétarien, et des conseils pour tenir le budget et faire de économies.

Ford a très vite compris l'importance de la communication. II crée rapidement un journal d'entreprise, Ford Times qui encourage les travailleurs à travailler dur et qui fait une critique acerbe des mauvais ouvriers qui gaspillent leur argent en boissons et sorties. En 1919, il rachète le Dearborn Independant, un journal local, mais qui tirera jusqu'à 700 mille exemplaires. II signe régulièrement des éditoriaux promouvant sans cesse l'idée d'une communauté d'intérêts dans l'industrie entre tous ceux qui y participent, qu'ils soient à la direction ou sur la chaîne.

\section{La stratégie managériale française: séduire et précariser subjectivement pour dominer}

En France, dans I'histoire récente, on distingue différentes phases qui constituent autant de tentatives de la part des employeurs d'opérer une métamorphose identitaire de leurs salariés pour les dominer. On distingue la période du «tout participatif», puis celle de la persuasion et de l'offensive éthique, adossé à celle de la précarisation subjective, qui précède celle de la prise en charge affective.

\subsection{La bataille participative}

Dans les années 80, les entreprises françaises, notamment les plus grandes d'entre elles, ont développé de multiples dispositifs participatifs. II faut dire que la période était particulière, les socialistes venaient d'arriver au pouvoir, et les lois Auroux (du nom du ministre du travail socialiste) visaient en 1982 à donner de nouveaux droits aux travailleurs. Parmi celles-ci, le droit d'expression direct et collectif leur donnait la possibilité d'émettre des vœux et avis sur les conditions et l'organisation de leur travail. Le patronat a tout d'abord crié "aux soviets», tout en se conformant à la loi et a vu assez rapidement qu'il pouvait être intéressant de mettre la hiérarchie en dialogue avec les salariés réunis au sein de leur collectif naturels. L'intention était de sortir d'un état endémique de confrontation, d'hostilité permanente pour faire advenir une situation plus consensuelle dans l'entreprise. L'idée d'instaurer une pacification interne via le dialogue, les échanges, et une certaine forme de coopération s'est ancrée dans l'esprit 
des responsables d'entreprise. L'enjeu était clairement d'amener les salariés à renoncer à leurs propres valeurs politiques, citoyennes et professionnelles (autour de questions aussi fondamentales que le travail bien fait, le travail utile, la possibilité de travailler seIon les règles du métier, les règles de l'art) et à adhérer à celles mises en œuvre par leur direction dans le cadre de ce qui s'annonçait comme une véritable guerre économique.

Cette bataille participative visait à harmoniser les points de vue des différents des protagonistes pour les aligner sur les critères et les valeurs de l'entreprise et à encadrer la politique d'individualisation qui dès le milieu des années 70 a été développée par le patronat en réaction aux événements de Mai 68 pendant lesquels eut lieu la plus longue grève générale du $20^{\mathrm{ème}}$ siècle. Le patronat s'était lancé dans une politique systématique d'individualisation de l'organisation du travail et de la gestion des salariés pour affaiblir les collectifs tout en prétendant répondre aux aspirations qui s'étaient manifestées au cours des grèves et manifestations (Boltanski \& Chiapello, 1998). Les salariés souhaitaient plus de liberté, d'autonomie, de respect, de dignité et de reconnaissance au travail, plus de responsabilités, ils voulaient s'épanouir dans leur activité professionnelle. C'est en surfant sur ces dimensions que les directions vont dérouler leur politique d'individualisation systématique et ont cherché à mettre en place les termes d'un consensus, e pour «moderniser» le rapport de leurs salariés au travail et à leur entreprise. Le discours mis en avant se résume dans la proclamation que la grande richesse des entreprises est constituée par les hommes et les femmes qui y travaillent et c'est en eux que résident les réserves de productivité. II faut leur donner la possibilité et les moyens de mobiliser leur intelligence, leur esprit d'initiative, au service de leur intérêt commun: la performance de leur entreprise. L'accent est mis sur la confiance, qui doit désormais réguler les relations entre directions, hiérarchies et salariés subalternes. Pendant les années 80 , un imposant dispositif participatif où voisineront groupes d'expression, cercles de qualité, et divers groupes ad hoc, se déroulera dans un grand nombre d'entreprises.

\subsection{L'émergence d'une éthique d'entreprise}

Les grandes entreprises se pensent en termes d'institutions, elles se pensent tout autant productrices de valeurs que d'organisation. Elles se lanceront au cours des années 90 dans la production de chartes éthiques, de codes déontologiques, de règles de vie, définissant les comportements et les manières de s'impliquer au travail, la nature des relations avec les collègues, la hiérarchie, les clients. Anne Salmon (2000; 2002) parle à juste titre d'offre éthique des entreprises, car celles-ci veulent proposer une nouvelle éthique définissant ce qu'est un salarié vertueux, un salarié qui ne pense pas qu'à ses intérêts égoïstes, mais prend en compte ceux de son entreprise qui rassemblent le bien commun. Il faut que les salariés acceptent de prendre sur eux, qu'ils visent l'excellence en permanence, qu'ils s'engagent à fond. 
Cette évolution attaque la place du travail dans notre société, le rôle qu'il y joue. La mise en place des nouvelles règles du jeu moral vise à redéfinir et à imposer un nouveau périmètre d'investissement subjectif des salariés. Les salariés sont appelés à s'investir dans le cadre des intérêts, de la philosophie et de la culture de leur entreprise, des ses critères d'efficacité professionnelle et comme on vient de le voir de son éthique. On assiste en fait à une volonté d'appropriation unilatérale du sens du travail, de sa finalité; une volonté de le désincarcérer de la société, c'est à dire d'instaurer une coupure du cordon ombilical qui relie chacun à la société à travers son travail.

Dans Le travail sans les autres (2009), je me suis efforcée d'analyser cette rupture instaurée dans le cadre de la modernisation managériale visant à produire le salarié idéal. Chaque salarié est supposé s'engager à fond, se mobiliser subjectivement dans le cadre défini strictement par l'entreprise et non plus dans celui constitué à partir de sa vision du monde, de sa morale, de ses valeurs professionnelles en relation avec les enjeux économiques, politiques de la société. Chacun est incité à travailler pour satisfaire les critères d'efficacité et de qualité choisis par l'entreprise. Cette rupture est rendue, du point de vue du management, nécessaire en raison surtout d'une série d'évolutions qui rendent les situations de travail plus fluctuantes et incertaines, et donc les formes antérieures d'organisation du travail moins pertinentes.

Nous nous trouvons là au cœur des tensions inscrites dans le contrat de travail salarial. L'organisation scientifique du travail de Taylor aboutissait à inscrire la contrainte et le contrôle dans la définition même des tâches, ce qui représentait un système de domination à la fois simple et efficace. L'organisation scientifique du travail plaçait l'emprise patronale au cœur de l'organisation du travail: les salariés n'avaient d'autre issue que de se conformer aux gestes et rythmes préconisés et véhiculées par l'organisation elle-même. C'était là une ressource précieuse pour l'employeur qui explique le succès planétaire et historique de cette forme d'organisation du travail.

Le taylorisme n'offre plus la même pertinence et efficacité, après sa remise en cause sociale, et en raison de l'évolution de la concurrence comme de la nature du travail. Celui-ci relève de plus en plus des activités tertiaires et notamment de service, ce qui désigne des situations de travail plus incertaines, instables, évolutives pour lesquelles une planification ex ante est souvent inappropriée et inopérante. Les directions se retrouvent, en fait, face à cette difficulté de dépendre à nouveau de l'état d'esprit de leurs salariés qui doivent se mobiliser subjectivement, cognitivement, affectivement aussi, pour trouver les solutions qui leur paraissent le mieux adaptées. Pour les directions, il est impératif qu'ils le fassent non pas selon leur point de vue professionnel, leur vision de la finalité, de l'utilité de leur travail, de leur appréciation de ce qu'est un travail de qualité, mais selon les consignes élaborées en fonction des critères très contextualisés de l'entreprise. 
D’où la volonté managériale de persuader, de convaincre les salariés de renoncer à leurs propres valeurs professionnelles, citoyennes, à leur morale pour relayer loyalement celles de leur entreprise. Le renoncement est d'ampleur, pour ces salariés, car il signifie un repositionnement dans le monde du travail, et l'alignement sur des logiques différentes de celles de leur métier, de leur morale, de leur valeurs.

\subsection{Une prise en charge de la vie intime}

Pour aider à ce renoncement, le management moderne tend à instiller un deal, non explicite mais néanmoins omniprésent, celui de la transaction narcissique. II s'agit de faire miroiter aux salariés la possibilité de se découvrir, de se surpasser et d'atteindre un idéal du moi en acceptant de relever les défis (l'excellence, l'engagement total) imposés par l'entreprise (de Gaulejac, 2005; Dujarier, 2006). Cette orientation ayant été largement préparée par l'individualisation, l'accompagnement participatif et la mise en concurrence des salariés. Envers les jeunes, cette orientation se pare d'accents plus ludiques, il s'agit de stimuler chez eux la recherche de l'aventure, de l'inconnu, l'esprit festif, le goût du paraître, l'esprit de compétition, la quête de soi, l'attirance pour le changement (Linhart, 2011).

Ces deux périmètres d'investissement subjectif, (la satisfaction narcissique et les intérêts de l'entreprise) sont interdépendants, car c'est de la conformité aux exigences de l'entreprise que découle la reconnaissance symbolique et matérielle destinée à nourrir l'implication narcissique. Mais ils sont déconnectés des enjeux de la société: chacun travaille pour soi et son entreprise et non plus pour prendre pied de façon citoyenne dans la société. Le travail tend de plus en plus à devenir une affaire personnelle et dépendant de la stratégie voulue par l'entreprise, il n'est plus une affaire de société. Comme le montre l'enquête de Baudelot et Gollac (2003), c'est individuellement et personnellement que chacun peut se sentir victime d'injustices voire d'exploitation. La dimension socialisatrice du travail est en passe d'être sérieusement «revisitée» dans le cadre de cette modernisation managériale, à la recherche de salariés conformes aux nouvelles règles du jeu du capitalisme mondialisé et financiarisé.

Dans cette optique, les frontières entre vie privée et vie professionnelle sont redéfinies par le management. II ne faut pas que la famille ou des sollicitations trop fortes de la vie extra professionnelle, viennent s'interposer et perturber la qualité de l'engagement dans le travail et notamment la disponibilité, la mobilité et la flexibilité. La mise en place de conciergeries d'entreprise vont dans ce sens d'une facilitation de la vie domestique, avec l'accès sur place à des crèches, des pressing, à des secrétariats (capables de régler des choses aussi diverses que des réservations pour les vacances, des places de théâtre, l'envoi d'un bouquet de fleurs, le développement de photo); et de la vie personnelle avec la mise à disposition de piscines, salles de relaxation, mas- 
sages.Les directions de ressources humaines revendiquent leur responsabilité dans ce domaine. Il leur revient d'aider les salariés à se maintenir en bonne forme, à ne pas importer leurs problèmes personnels dans leur entreprise et dans leur travail. Au cours d'une rencontre organisée par un club $\mathrm{RH}$ réunissant des responsables Ressources Humaines de grandes entreprises, à laquelle j'ai pu assister, j'ai été frappée de voir que des militaires (deux généraux, un amiral et un colonel) avaient été invités comme intervenants à présenter leur politique $\mathrm{RH}$ qui semblait passionner d'ailleurs les participants. II n'est pas inintéressant de relater une anecdote qui m’a paru révélatrice de l'ampleur de l'intrusion managériale dans la vie privée, intime des salariés, en l'occurrence des soldats dont l'exemple fascinait les responsables RH présents. Un amiral expliquait que lorsque des marins embarquent pour des campagnes de plusieurs mois à bord d'un sous-marin ou d'un bateau, un problème préoccupant pouvait venir de pensées perturbantes quant à la fidélité de leur épouse pendant cette longue période. Ces pensées pouvaient obséder les marins au point de perturber l'efficacité de leur activité et mettre en péril leur vigilance, elle pouvait nuire à l'ambiance aussi. Or, un des moments les plus délicats pour la fidélité des épouses résidait, dixit l'amiral, dans le moment où un appareil ménager tombait en panne ce qui exigeait la venue, au domicile de la dame, d'un dépanneur. La sollicitude du responsable RH allait jusqu'à prévoir un service de dépannage, contrôlé par les soins des RH, de sorte qu'il n'y ait pas à s'inquiéter que des difficultés de la vie pratique puissent déboucher sur des situations à risques (un plombier ou un électricien qui ferait des avances à madame). C'est cette orientation que les cabinets de consultants nomment le management bienveillant. II faut que les salariés soient entièrement disponibles psychiquement et matériellement lorsqu'ils sont au travail, délestés des soucis de la vie domestique et familiale, mais aussi des frustrations liées à la perte d'un sens personnalisé de leur travail et des valeurs qu'ils auraient aimé y inscrire.

Certaines entreprises prennent aussi en charge le besoin de don, d'altruisme dont certains salariés peuvent être frustrés en raison de l'orientation très productiviste et rentabiliste de leur entreprise dans le cadre d'un capitalisme de plus en plus financier. Certaines directions organisent alors des actions humanitaires où les salariés sont invités à se mobiliser dans le cadre de congés dédiés aux actions solidaires, susceptibles de satisfaire de valeurs d'utilité sociale. La loi (du 25 janvier 2012) relaie également des formes de générosité en entreprise, en cadrant le don de RTT de salariés à un de leur collègue qui aurait un enfant ou un parent gravement malade. ${ }^{2}$ II s'agit de générosité qui trouve à s'exprimer dans l'entreprise mais dans le cadre d'action périphérique au travail lui-même.

L'éthique du travail que le management cherche à promouvoir diffuse une représentation nouvelle des enjeux qui les synthétise dans l'apologie d'un engagement fort pour ne pas dire total dans le travail selon les critères d'efficacité définis par la direction.
2. Selon la loi dite du don de Réduction du temps de travail (RTT), du 25 janvier 2012, "un salarié peut, sur sa demande et en accord avec l'employeur, renoncer anonymement à tout ou partie de ses jours de repos non pris [...] au bénéfice d'un autre salarié de l'entreprise qu assume la charge d'un enfant âgé de moins de 20 ans atteint d'une maladie, d'un handicap ou victime d'un accident d'une particulière gravité rendant indispensables une présence soutenue et des soins contraignants". 
Pour ce faire, le management est prêt à s'investir dans la gestion des besoins matériels et spirituels de leurs salariés hors de l'activité de travail elle-même qui doit se dérouler de la façon jugée la plus efficace par les directions. En d'autres termes, le salarié est appelé à se mobiliser entièrement, en toute liberté d'esprit, pour trouver comment faire l'usage de lui-même le plus rentable (Schwartz, 1997) dans l'optique voulue par son employeur. II ne doit importer ni ses problèmes, ni ses problématiques (valeurs professionnelles, citoyennes), mais s'abandonner à celles de son entreprise.

Mais toutes ces pratiques managériales censées préparer les conditions de l'émergence d'un salarié parfaitement adapté au modèle d'organisation ne peuvent suffire à elles seules. II faut dans l'urgence s'assurer que les salariés soient mis en situation de se conformer strictement à ce que l'on attend d'eux, même s'ils ne sont pas encore acquis à la cause de leur entreprise et restent influencés par des schèmes de pensées, des valeurs et des intérêts relevant d'une autre vision du monde.

Pour ce qui concerne l'organisation du travail lui-même, on sort résolument de la posture de bienveillance. Les politiques mises en œuvre relèvent de logiques de déstabilisation, de vulnérabilisation, de fragilisation des salariés au travail qui expliquent le malaise, le mal-être voire la souffrance au travail relayés par les médias.

Ces politiques reflètent la conviction managériale que pour disposer de salariés adaptés aux exigences de la concurrence économique, il faut - en attendant les effets des stratégies de persuasion, séduction, bienveillance - opérer une forte pression sur eux. Il s'agit de les mettre dans une situation qui ne leur laisse pas d'autre choix que de s'adosser aux consignes, exigences des directions, d'adopter les manières d'agir, de travailler qu'elles préconisent selon les critères qu'elles valident. Le management n'a pas lésiné sur les efforts (démarches participatives, offre éthique, bienveillance des $\mathrm{DRH}$ ) destinés à créer chez ses salariés une autre approche des enjeux du travail. Mais cela ne garantit pas que la bataille identitaire soit gagnée. Elle est incontestablement toujours en cours. En attendant que les choses mûrissent, que les générations évoluent, il faut parer au plus pressé. Prendre des mesures plus immédiatement efficaces qui permettront de mobiliser de façon rentable des salariés pas tout à fait convaincus, ni totalement séduits. On voit cette orientation à l'œuvre dans la plupart des grandes entreprises et de façon plus éclatante encore dans le secteur public, où il s'agit de forcer le chemin de la loi organique relative aux lois de finances (Lolf) et de la révision générale des politiques publiques (RGPP) qui heurtent de plein fouet l'éthique professionnelle de nombreux agents. 


\subsection{La précarisation subjective des salariés: le va-tout managérial}

On connait la précarisation objective par les emplois - interim, temps partiel subi, contrat à durée déterminée (CDD), travail saisonnier, stages - et ses effets sur les comportements professionnels des salariés qui la subissent. S'ils veulent accéder un jour à cet Eldorado que représente pour eux un emploi stable dans l'entreprise où ils se trouvent, il leur faut faire, en permanence, la démonstration de leurs qualités professionnelles et surtout de leur bonne volonté. Ils ont intérêt à se présenter comme des salariés fiables et conformes à ce qu'on attend d'eux; c'est là une situation propice du point de vue du management car ces salariés ne vont pas chercher à contrecarrer les manières de travail qu'il veut imposer. Même ces emplois précaires tendent à se développer et à concerner des salariés de plus en plus nombreux et notamment les jeunes qui transitent de plus en plus souvent pas ce type d'emplois, ils ne concernent pas, loin de là, la majorité des salariés, protégés en tant que détenteurs d'un contrat à durée indéterminée (CDI) ou fonctionnaires, par un code du travail ou de la fonction publique, relativement favorable.

On pourrait résumer ce recours managérial à la précarisation subjective par le choix d'une mise sous tensions, d'une fragilisation des salariés, en vue de leur retirer les repères, les appuis, les ressources susceptibles de leur ménager une certaine liberté d'esprit, une assurance professionnelle leur permettant d'opposer à la hiérarchie un autre point de vue sur la manière de travailler, et de le concrétiser par des pratiques qu'ils jugent plus adaptées à leurs propres valeurs.

Il s'agit d'assiéger concrètement les salariés, pour qu'ils rendent les armes, et fassent acte de soumission subjective à la rationalité de l'entreprise, à ses critères d'efficacité, de rentabilité, et donc à ses critères de qualité. Cette offensive a en ligne de mire l'expérience des salariés et les métiers, deux piliers fondamentaux qui confèrent aux salariés la capacité de maîtriser, de dominer leur travail, de s'affirmer à travers lui, et leur confèrent une légitimité à vouloir se faire entendre, à ne pas subir des ordres, des consignes, des objectifs qui leur paraîtraient en décalage avec les règles du métier, les règles de l'art, les connaissances accumulés dans la fonction ou le poste. Deux piliers qui peuvent fusionner d'une certaine façon (le métier ne représente-t-il pas une expérience collective, validée, certifiée et légitimée, une expérience collective coagulée?) et auxquels viennent se fondre l'aide des autres, inscrits dans les réseaux professionnels informels où chacun peut trouver des personnes ressources.

Pour arracher la reddition subjective, c'est à dire, le consentement des salariés, obtenir de leur part l'engagement et le dévouement recherchés, dans le cadre des critères et modes de travail impérativement voulues par les directions, le choix de la déstabi- 
lisation systématique par le changement permanent et la mobilité systématique s'observe dans nombre d'entreprises modernes du secteur privé comme public. C'est ainsi que l'on peut analyser ce management moderne qui pratique une politique systématique de changements, et de mobilités de toutes natures: mobilité professionnelle et géographique des salariés et de leurs cadres, réformes, redéfinition, recomposition des métiers, externalisation de certaines activités qui seront plus tard à nouveau reprises, fusion de différentes natures, déménagements, délocalisations relocalisations géographiques etc. (Linhart, 2011). Certes, il y a à l'évidence, une volonté de s'adapter à un environnement mouvant, et à des enjeux court termistes, mais il y a, de façon toute aussi évidente, l'idée que le changement en soi est une bonne chose: II est devenu, aux yeux du management, une vertu en soi qui se substitue à la notion de progrès comme l'analyse Anne Salmon (2000).

Dépouiller les salariés de leur expérience, c'est les mettre à découvert, les faire évoluer au sein de leur activité sans filet, sans boussole, sans appui, en permanence sur le fil du rasoir, déstabilisés et dans la nécessité de se raccrocher in extremis aux logiques émanant de son entreprise. Du côté managérial, cette expérience individuelle et collective est disqualifiée et présentée sous la forme de routines, habitudes, acquis. Elle est assimilée à des rigidités, archaïsmes (dans le contexte d'un environnement de plus en plus incertain), et parfois même à des signes de paresse intellectuelle, de refus de prises de risques, d'absence de courage. En tous cas, elle est en permanence identifiée comme frein à l'innovation, au progrès, à l'efficacité. Comme le refus de se comporter en véritable acteur de l'entreprise. La mobilité systématique et les restructurations sont là pour secouer les salariés («il nous faut en permanence secouer le cocotier» m'avait dit dans les années 90 un manager d'une grande entreprise), les délester de l'expérience, des règles du métier, et des autres, de ceux qui partageant un même destin professionnel pourraient les conforter et renforcer (Linhart, 2009).

De fait, les salariés modernes ne se sentent pas chez eux, ni entre eux, dans leur entreprise, dans leur travail. Ils s'y sentent comme des étrangers, dans un environnement hostile, ils ont en permanence à réapprendre, à s'adapter, à réinventer les modalités nécessaires pour maîtriser leur activité: savoir qui peut être une personne ressource, quelles relations peuvent être établies avec les différents services, ou interlocuteurs, comment se conforter dans la décision qu'ils prennent puisqu'ils n'ont plus le recul nécessaire. À réinventer des routines, car ces routines permettent de fonctionner à l'économie pour se consacrer efficacement aux incidents, aux imprévus dans un contexte qui devient plus complexe et plus incertain.

Avec cette politique de réformes systématiques, les individus au travail sont en situation permanente de désapprentissage et réapprentissage (Metzger, 1999). La précarisation subjective, ce n'est pas seulement la peur d'être conduit un jour à la faute 
professionnelle qui peut faire perdre son emploi, mais c'est aussi une mise en danger de soi, par le risque de se trouver dans une situation de quasi incompétence qui porte atteinte au sentiment de valeur, de dignité, de légitimité. Les salariés sont artificiellement mis en état de fébrilité, d'alerte, d'inquiétude, ce qui est l'objectif recherché pour obtenir d'eux qu'ils développent précisément les compétences, l'excellence, l'engagement configurés selon les besoins et les valeurs de leur entreprise. Pour qu'ils s'approchent de la figure du travailleur convoité par le management.

Bouger sans arrêt les personnes, les organisations, les structures et les procès, effacer les repères, bousculer les habitudes, c'est maintenir les individus dans un état d'insécurité, de vulnérabilité censé les pousser à reconfigurer leurs registres affectifs et cognitifs pour s'ajuster à ceux qui correspondent à la rationalité de leur entreprise; à s'en remettre aux règles du jeu de cette dernière, à faire confiance à leur direction. Mais cela devient vite une source de mal-être et de souffrance, dès lors où cela va de pair avec une réduction des effectifs, un raccourcissement des délais, pour des charges de travail qui augmentent. Or l'intensification quasi systématique du travail est devenue désormais une donnée de la modernisation.

Au sein du travail lui-même, la modernisation a planté les germes d'une insécurité généralisée, destinée à faire émerger un type de salarié conforme aux exigences d'un management qui se fonde sur la méfiance, la défiance a priori à l'égard de ceux qu'il emploie. L'entretien individuel, pratiqué nombreuses grandes entreprises, au cours duquel chaque salarié se voit fixer, par son supérieur immédiat, ses objectifs personnels et évaluer sa performance chaque année, est une cause d'angoisse qui illustre bien ce phénomène. En raison de leur forte mobilité, les responsables ne sont en général pas des spécialistes des métiers qu'ils ont à évaluer, ce sont des managers: nombre de salariés rapportent l'état d'ignorance dans lequel se trouvent les responsables évaluateurs, de la réalité des situations de travail qu'ils ont à évaluer, leur incompréhension des contraintes qui pèsent sur les personnes qu'ils ont à juger, à noter. Or ces entretiens ont des implications bien réelles sur les primes, les formations à suivre, les déroulements de carrière et parfois même sur la permanence dans l'entreprise. Les salariés disent appréhender ces moments où ils se sentent jaugés, jugés, sans que les bases de cette évaluation ne soient clairement établies et justifiées. Un sentiment de précarité s'installe lorsque se développe la conviction d'être confronté à des jugements aléatoires ou arbitraires. Leur sort dépend de responsables qui ne connaissent pas leur vrai travail. Ils disent alors avoir le sentiment d'être piégés, et appréhendent les périodes d'entretiens qui peuvent décider éventuellement de leur éviction.

Le sentiment d'abandon que peuvent éprouver les salariés ressort de nombre d'enquêtes le terrain. Les salariés affirment ne pas trouver de soutien ou d'aide lorsqu'ils 
se trouvent confrontés aux tensions organisationnelles, et à des objectifs contradictoires. La hiérarchie est indisponible ou non compétente et ils doivent trouver euxmêmes des solutions aux multiples défis qui s'accumulent. Ils se trouvent parfois acculés à faire du mauvais travail. II en ressort une angoisse permanente, un sentiment d'incompréhension voire d'une perte de sens.

Abstract: This article aims at developing the idea that each new capitalistic labour process produces an ideological and ethical rhetoric so as to legitimate a form that appears to be contradictory to the essence of political democracies, which is characterized by the fact that no one belongs to another. Taylor and Ford produced an important ideological effort to promote the allegation that no conflict of interests exists between workers and employers while they attacked systematically the workers resources within the forces struggle. The French modern managerial system follows the same path, leading an attempt to seduce and convince the wage earners on the one hand, though developing a strategy of subjective precarization on the other hand by a policy of perpetual changes in order to destabilize them.

Keywords: Taylor, Ford, ideology, new management, subjective precarization.

\section{Bibliographie}

BAUdELOT, Chritian; GolLAC, Michel et alii. Faut-il travailler pour être heureux? Le bonheur et le travail en France. Paris: Fayard: 2003.

BoltANSKI, Luc; ChIAPELLO, Eve. Le nouvel esprit du capitalisme. Paris: NRF, Essais Gallimard, 1999.

Davolne, Lucie; MedA, Dominique. Place et sens du travail en Europe; une singularité française. CEE-Document de Travail, n. 96-1, 2008.

DujARIer, Marie-Anne. L'idéal du travail. Paris: Le Monde; Presses Universitaires de France, 2006.

FORD, Henry. "My philosophy of industry», an authorized interview by Fay Leone Faurote. New York: Coward-Mc Cann, 1929.

— . Ma vie, mon œuvre. Paris: Payot, 1925.

_. Propos d'hier et d'aujourd'hui. Paris: Masson, 1992 [1926].

FOSTER, John. The fetish of Henry Ford. New York: Monyhly Review, 1988. Problèmes Économiques, n. 2093, 5 Oct. 1988.

GaUlejAC, Vincent (de). Travail, les raisons de la colère. Patis: Le Seuil, 2011.

— . La société malade de la gestion. Paris: Le Seuil, 2005.

IRIBARne, Philippe (d'). La logique de l'honneur. Gestion des entreprises et stratégies nationales. Paris: Le Seuil, 1989. 
LINHART, Danièle. Le management moderne, un modèle conçu pour les jeunes? Vultur et Mercure, «Perspectives internationales sur les jeunes». Québec: Presses de l'Université de Laval, 2011.

—. Une précarisation subjective du travail? Réalités Industrielles, Annales des Mines, Fév. 2011.

— . La modernisation des entreprises. Paris: La Découverte,2010.

_. Travailler sans les autres? Paris: Le Seuil, 2009.

_. Perte d'emploi, perte de soi, nouvelle version. Toulouse: Erès, 2009 (avec la collaboration de B. Rist et E. Durand).

_ . Le torticolis de l'autruche. L'éternelle modernisation des entreprises. Paris: Le Seuil, 1991.

LINHART, Danièle; MAUCHAMP, Nelly. Le travail. Paris: Le cavalier Bleu, 2009.

MetZGER, Jean Luc. Entre utopie et résignation: la réforme permanente d'un service public. Paris: L'Harmattan, 2000.

TAYLOR, Frederick Winslow. La direction scientifique des entreprises. Paris: Dunod, 1956 [1912].

SALMON, Anne. La tentation éthique du capital. Paris: Éditions du CNRS, 2000.

- Éthique et ordre économique, une entreprise de séduction. Paris: Éditions du CNRS, 2002.

SALMON, Christian. Story telling. Paris: La Découverte, 2007.

SCHWARTZ, Yves. Reconnaissance du travail. Pour une approche ergologique. Paris: Presses Universitaires de France, 1997.

SWARD, Keith. The legend of Henry Ford. New York: Russel \& Russel, 1968. 